\title{
A 15-year-old boy with sickle cell disease, chest pain and respiratory distress
}

\author{
Yves D. Pastore MD, Nancy Robitaille MD, Veronique Naessens MD; for the Canadian Haemoglobinopathy \\ Association (CanHaem)
}

Cite as: CMAJ 2017 December 11;189:E1529-30. doi: 10.1503/cmaj.170526

A 15-year-old boy with homozygous S sickle cell disease presents to the emergency department with chest pain and low-grade fever three days after he had a mild vasoocclusive crisis that was treated with ibuprofen and morphine. His oxygen saturation level is $95 \%$ in room air, his blood pressure is normal, he has a heart rate of 126 beats/min, and his body temperature is $38.1^{\circ} \mathrm{C}$. Chest auscultation shows rales on the right lower side. A complete blood cell count analysis reports a hemoglobin level of 68 (normal range 12.9-16.7) g/L, absolute reticulocyte count of 365 (normal range 50-150) $\times 10^{9} / \mathrm{L}$, leukocyte count of 18 (normal range according to age and ethnicity $3-9.7$ ) $\times 10^{9} / \mathrm{L}$ and a platelet count of 486 (normal range 165$360) \times 10^{9} / \mathrm{L}$. Chest radiography confirms an infiltrate in the lower lobe of the right lung.

\section{Does this patient have acute chest syndrome?}

Acute chest syndrome is an acute complication of sickle cell disease that is defined by a new pulmonary infiltrate, but not atelectasis, on chest radiography, and at least one other symptom, such as fever, chest pain, or respiratory signs such as tachypnea, wheezing or cough. ${ }^{1,2}$ Clinicians should have a high index of suspicion for acute chest syndrome, because it is a leading cause of admission to hospital and death among patients with sickle cell disease ${ }^{1,2}$ (Box 1).

Chest pain or respiratory symptoms in patients with sickle cell disease should prompt an evaluation for acute chest syndrome. ${ }^{2}$ The cause of acute chest syndrome is generally multifactorial: infections, fat embolization from infarcted bone marrow or intravascular pulmonary sequestration of sickled red blood cells. ${ }^{3,4}$

\section{Are investigations needed?}

According to the consensus statement from the Canadian Haemoglobinopathy, ${ }^{2}$ a chest radiograph is required to diagnose acute chest syndrome. Multilobe involvement has been associated with increased risk of respiratory failure. Baseline laboratory tests should be obtained (Box 1). Cross-matched units of red blood cells should be secured early on admission to hospital, because blood transfusion should be considered if the patient's respiratory condition worsens. In these patients, analysis of arterial or capillary blood gases may also help to assess the severity of hypoxemia. 2,5
Investigation for an infectious process, including respiratory viruses, Mycoplasma pneumoniae and Chlamydophila pneumoniae, is recommended because they are associated with acute chest syndrome. ${ }^{1}$ Fever in a patient with acute chest syndrome should prompt blood cultures and the start of antibiotics administered intravenously that include atypical coverage (e.g., cefotaxime and azithromycin).

\section{Should this patient be admitted to hospital?}

Because acute chest syndrome can rapidly deteriorate, patients with suspected acute chest syndrome should be admitted to hospital. It may be prudent to notify the intensive care unit of the admission of a patient with acute chest syndrome, because ventilatory support may be required. Indications for blood transfusion include worsening respiratory condition or a decrease in hemoglobin level of more than $20 \mathrm{~g} / \mathrm{L}$ below baseline. ${ }^{1,2,4,5}$ Units

Box 1: Summary of recommendations on managing acute chest syndrome ${ }^{2}$

Acute chest syndrome (ACS) is a leading cause of admission to hospital and death among children and adults with sickle cell disease. Clinicians should be aware of the following:

- Have a high index of suspicion for ACS.

- Admit patients with suspected ACS to hospital for close care and monitoring.

- Administer high-flow oxygen to maintain an oxygen saturation level of $95 \%$ or higher.

- Maintain hydration using intravenous and oral intake to keep total fluid intake at maintenance rate.

- Obtain a complete blood cell count, arterial blood gases and extended cross-match for possible exchange or simple transfusion.

- Consider transfusion in most patients with ACS; for patients with severe ACS or a rapidly progressing condition, exchange transfusion should be considered.

- Obtain blood culture from febrile patients and treat with broad spectrum antibiotics, including a macrolide, because Mycoplasma pneumoniae and Chlamydophila pneumoniae can underlie ACS.

- Do not use corticosteroids routinely. 
of red blood cells that have been phenotypically matched for C, E and Kell antigens should be given to reduce the risk of alloimmunization. ${ }^{6}$ In addition, the posttransfusion hemoglobin level should not exceed 100 to $110 \mathrm{~g} / \mathrm{L}$, because a higher threshold in patients with sickle cell disease has been associated with risk of stroke. ${ }^{7}$ Therefore, if the patient has a hemoglobin level of $90 \mathrm{~g} / \mathrm{L}$ or more, an exchange transfusion should be used instead of a simple transfusion. ${ }^{6}$ Similarly, an urgent exchange of red blood cells is preferred for any patient with a rapidly deteriorating respiratory status, especially if mechanical support is required. ${ }^{5}$

Bronchodilator therapy should be given to patients with evidence of reactive airway disease. Routine use of corticosteroids is not recommended, unless there is clear evidence of an association with asthma exacerbation. ${ }^{2}$

\section{Case revisited}

The patient with acute chest syndrome was admitted to hospital and the hematology service was consulted. Given that this patient had infiltrate in the lung and low-grade fever, we started treatment with ceftriaxone administered intravenously along with azithromycin taken orally. ${ }^{2,4,5}$ We started intravenous hydration with normal saline at a maintenance rate, along with morphine and a nonsteroidal anti-inflammatory agent for pain control when required.

Eight hours after the patient was admitted to hospital, his oxygen saturation level decreased to $91 \%$ at room air, and he developed mild grunting. Given the clinical deterioration, we started oxygen supplementation and transfused one unit of packed red blood cells that were phenotypically matched for C, E and Kell antigens. ${ }^{2,4,5}$ Within the next 48 hours, he gradually improved and he was weaned off oxygen supplementation.

After five days in hospital, the patient was discharged on high-dose amoxicillin. We stopped treatment with azithromycin after polymerase chain reaction testing was negative for M. pneumoniae.

\section{References}

1. Yawn BP, Buchanan GR, Afenyi-Annan AN, et al. Management of sickle cell disease: summary of the 2014 evidence-based report by expert panel members. JAMA 2014;312:1033-48.

2. The consensus statement on the care of patients with sickle cell disease in Canada. CanHaem; 2015. Available: http://canhaem.org/the-consensus-statement -on-the-care-of-patients-with-sickle-cell-disease-in-canada/ (accessed 2017 Apr. 17).

3. Gladwin MT, Vichinsky E. Pulmonary complications of sickle cell disease. N Engl J Med 2008;359:2254-65.

4. Miller ST. How I treat acute chest syndrome in children with sickle cell disease. Blood 2011;117:5297-305.

5. Howard J, Hart N, Roberts-Harewood M, et al. BCSH Committee. Guideline on the management of acute chest syndrome in sickle cell disease. Br J Haematol 2015;169:492-505.

6. Chou ST. Transfusion therapy for sickle cell disease: a balancing act. Hematology (Am Soc Hematol Educ Program) 2013;2013:439-46.

7. Castro O, Brambilla DJ, Thorington B, et al. The acute chest syndrome in sickle cell disease: incidence and risk factors. The Cooperative Study of Sickle Cell Disease. Blood 1994;84:643-9.
Competing interests: Yves Pastore has received personal fees from Pfizer, Novartis and ApoPharma, outside the submitted work. Veronique Naessens has received personal fees in the form of renumeration for participation on the advisory boards of Pfizer and Alexion, and lecture fees from ApoPharma. The Canadian Haemoglobinopathy Association received unrestricted educational funds from ApoPharma, Novartis and Pfizer to support production of the consensus statement. No other competing interests were declared.

This article has been peer reviewed.

The clinical scenario is fictional.
Affiliations: Hematology Service (Pastore, Robitaille), Department of Pediatrics, CHU Sainte-Justine, Université de Montréal; Division of Hematology (Naessens), McGill University Health Centre, Montréal, Que.

Contributors: Yves Pastore designed the work and drafted the manuscript. Nancy Robitaille and Veronique Naessens contributed to the drafting and revision of the manuscript. All of the authors revised the manuscript critically for important intellectual content, gave final approval of the version to be published and agreed to be accountable for all aspects of the work.
Acknowledgements: The authors would like to thank Dr. Madeleine Verhovsek for her critical review of the manuscript. This work was performed on behalf of the Canadian Haemoglobinopathy Association (http://canhaem.org).

Correspondence to: Yves Pastore, yves. pastore@umontreal.ca 